\title{
Topics (Disinformation)
}

\section{AUTHOR}

Anna Staender, Edda Humprecht

\section{KEYWORDS}

topics, disinformation, sources, misinformation, conspiracy theories, social media

\section{BRIEF DESCRIPTION}

The topic variable is used in research on disinformation to analyze thematic differences in the content of false news, rumors, conspiracies, etc. Those topics are frequently based on national news agendas, i.e. producers of disinformation address current national or world events (e.g. elections, immigration, etc.) (Humprecht, 2019).

FIELD OF APPLICATION/THEORETICAL FOUNDATION Topics are a central yet under-researched aspect of research on online disinformation (Freelon \& Wells, 2020). The research interest is to find out which topics are taken up and spread by disinformation producers. The focus of this research is both on specific key topics for which sub-themes are identified (e.g. elections, climate change, Covid-19) and, more generally, on the question of which misleading content is disseminated (mostly on social media). Methodologically, the identification of topics is often a first step followed by further analysis of the content (Ferrara, 2017). Thus, the analysis of topics is linked to the detection of disinformation, which represents a methodological challenge. Topics can be identified inductively or deductively. Inductive analyses often use a data corpus, for example social media data, and try to identify topics using techniques such as topic modelling (e.g. Boberg et al., 2020). Deductive analyses frequently use topic lists to classify contents. Topics lists are initially created based on the literature on the respective topic or with the help of databases, e.g. by fact-checkers.

\section{REFERENCES/COMBINATION WITH OTHER} METHODS OF DATA COLLECTION

Studies on topics of disinformation use both manual and automated content analysis or combinations of both to investigate the occurrence of different topics in texts (Boberg et al., 2020; Bradshaw, Howard, Kollanyi, \& Neudert, 2020). Inductive and deductive approaches have been combined with qualitative text analyses to identify topic categories which are subsequently coded (Humprecht, 2019; Marchal, Kollanyi, Neudert, \& Howard, 2019).

\section{EXAMPLE STUDIES:}

Ferrara (2017); Humprecht (2019); Marchal et al. (2019) 
Table 1. Summary of selected studies.

\begin{tabular}{|c|c|c|c|}
\hline Author(s) & Sample & Values & Reliability \\
\hline $\begin{array}{l}\text { Ferrara } \\
(2017)\end{array}$ & $\begin{array}{l}\text { Content type: Tweets } \\
\text { Sampling period: April 27, } 2017 \text { to } \\
\text { May 7, } 201 \\
\text { Sample size: } 16.65 \text { million tweets } \\
\text { Sampling: List of } 23 \text { key words } \\
\text { and top } 20 \text { hashtags }\end{array}$ & $\begin{array}{l}\text { Keywords: France2017, Ma- } \\
\text { rine2017, AuNomDuPeuple, } \\
\text { FrenchElection, FrenchElec- } \\
\text { tions, Macron, LePen, Mari- } \\
\text { neLePen, FrenchPresidentia- } \\
\text { lElection, JeChoisisMarine, } \\
\text { JeVoteMarine, JeVoteMacron } \\
\text { JeVote, Presidentielle2017, } \\
\text { ElectionFracaise, JamaisMa- } \\
\text { cron, Macron2017, EnMarche, } \\
\text { MacronPresident } \\
\text { Hashtags: } \\
\text { \#Macron, \#Presidentielle2017, } \\
\text { \#fn, \#JeVote, \#LePen, \#France, } \\
\text { \#2017LeDebat, \#MacronLeaks, } \\
\text { \#Marine2017, \#debat2017, } \\
\text { \#2017LeDébat, \#MacronGate, } \\
\text { \#MarineLePen, \#Whirlpool, } \\
\text { \#EnMarche, \#JeVoteMacron, } \\
\text { \#MacronPresident, \#JamaisMa- } \\
\text { cron, \#FrenchElection }\end{array}$ & - \\
\hline $\begin{array}{l}\text { Hum- } \\
\text { precht } \\
\text { (2019) }\end{array}$ & $\begin{array}{l}\text { Content type: fact checks } \\
\text { Outlet/ country: } 2 \text { fact checkers } \\
\text { per country (AT, DE, UK, US) } \\
\text { Sampling period: June 1, } 2016 \text { to } \\
\text { September } 30,2017 \\
\text { Sample size: N=651 } \\
\text { Unit of analysis: story/ fact-check } \\
\text { No. of topics coded: main topic per } \\
\text { fact-check } \\
\text { Level of analysis: fact checks and } \\
\text { fact-checker }\end{array}$ & $\begin{array}{l}\text { conspiracy theory, education, } \\
\text { election campaign, environ- } \\
\text { ment, government/public } \\
\text { administration (at the time } \\
\text { when the story was published), } \\
\text { health, immigration/integra- } \\
\text { tion, justice/crime, labor/em- } \\
\text { ployment, macroeconomics/ } \\
\text { economic regulation, media/ } \\
\text { journalism, science/ technolo- } \\
\text { gy, war/terror, others }\end{array}$ & $\begin{array}{l}\text { Krippendorff's } \\
\text { alpha }=0.71\end{array}$ \\
\hline
\end{tabular}




\begin{tabular}{ll} 
Author(s) & Sample \\
\hline Marchal & Content type: tweets related to the \\
(2019) & European elections 2019 \\
& Sampling: hashtags in English, \\
& Catalan, French, German, Itali- \\
& an, Polish, Spanish, Swedish \\
& Sampling criteria: (1) contained at \\
& least one of the relevant hash- \\
& tags; (2) contained the hashtag \\
& in the URL shared, or the title of \\
& its webpage; (3) were a retweet \\
& of a message that contained a \\
& relevant hashtag or mention in \\
& the original message; (4) were \\
& a quoted tweet referring to a \\
& tweet with a relevant hashtag or \\
& mention \\
& Sampling period: 5 April and 20 \\
& April, 2019 \\
& Sample size: 584,062 tweets from \\
& 187,743 unique users
\end{tabular}

Religion Islam (Muslim, Islam, Hijab, Halal, Muslima, Minaret) Religion Christianity (Christianity, Church, Priest) Immigration (Asylum Seeker, Refugee, Migrants, Child Migrant, Dual Citizenship, Social Integration)

Terrorism (ISIS, Djihad, Terrorism, Terrorist Attack)

Political Figures/Parties (Vladimir Putin, Enrico Mezzetti, Emmanuel Macron, ANPI, Arnold van Doorn, Islamic Party for Unity, Nordic Resistance Movement) Celebrities (Lara Trump, Alba Parietti)

Crime (Vandalism, Rape, Sexual Assault, Fraud, Murder, Honour Killing)

Notre-Dame Fire (Notre-Dame Fire, Reconstruction) Political Ideology (Anti-Fascism, Fascism, Nationalism) Social Issues (Abortion, Bullying, Birth Rate)

\section{REFERENCES}

Boberg, S., Quandt, T., Schatto-Eckrodt, T., \& Frischlich, L. (2020). Pandemic Populism: Facebook Pages of Alternative News Media and the Corona Crisis -- A Computational Content Analysis, 2019. Retrieved from http://arxiv.org/abs/2004.02566

Bradshaw, S., Howard, P. N., Kollanyi, B., \& Neudert, L. M. (2020). Sourcing and Automation of Political News and Information over Social Media in the United States, 2016-2018. Political Communication, 37(2), 173-193. https:// doi.org/10.1080/10584609.2019.1663322

Ferrara, E. (2017). Disinformation and social bot operations in the run up to the 2017 French presidential election. First Monday, 22(8). https://doi.org/10.5210/FM.V22I8.8005

Freelon, D., \& Wells, C. (2020). Disinformation as Political Communication. Political Communication, 37(2), 145-156. https:// doi.org/10.1080/10584609.2020.1723755

Humprecht, E. (2019). Where 'fake news' flourishes: a comparison across four Western democracies. Information Communication and Society, 22(13), 1973-1988. https:// doi.org/10.1080/1369118X.2018.1474241

Marchal, N., Kollanyi, B., Neudert, L., \& Howard, P. N. (2019). Junk News During the EU Parliamentary Elections : Lessons from a Seven-Language Study of Twitter and Facebook. Oxford, UK. Retrieved from https:// comprop.oii.ox.ac.uk/wp-content/uploads/ sites/93/2019/05/EU-Data-Memo.pdf 